\title{
Considerazioni sulla tecnica della puntura ad occhiello
}

\section{Infermieri del CAL Dialisi di Mariano Comense (Como)}

Nel CAL Dialisi di M ariano Comense (CO) dializziamo 24 pazienti al giorno articolati su due turni con 4 infermieri per turno.

Nel luglio 2006 a seguito di un incontro di formazione sugli accessi vascolari è stata presentata una relazione sulla "puntura ad occhiello", tecnica già in uso in altri Centri dialisi italiani e di Paesi europei.

Da luglio 2006 fino a gennaio 2007 circa, questa tecnica è stata usata in un solo paziente che presentava dolore intenso durante la venipuntura, successivamente si è utilizzata per altri 8 pazienti che presentavano:

1) dolore alla puntura delle FAV;

2) FAV di recente confezionamento (per evitare la formazione di aneurismi);

3) difficoltà nel reperire i vasi da incannulare.

L' équipe di lavoro, comprendeva tutto il personale infermieristico, che mantenendo i criteri di scelta del sito di puntura, ha permesso la creazione di un "cono cicatriziale" senza grosse difficoltà.

La procedura della puntura ad occhiello prevedeva, dopo la formazione del cono cicatriziale, l'utilizzo di aghi non taglienti (smussi), ma questo nel nostro Centro non è mai avvenuto (se non occasionalmente con I'utilizzo di qualche campione) in quanto gli aghi non erano disponibili fino a marzo 2008.

Durante l'utilizzo di questi ultimi i pazienti hanno riferito una diminuzione del dolore durante la venipuntura e gli operatori non hanno segnalato nessuna difficoltà nel loro inserimento.

Inizialmente si sono riscontrate (in alcuni pazienti) delle difficoltà nella rimozione della crosta prima della venipuntura e il sanguinamento intradialitico, problematiche regredite con il tempo.

I vantaggi nell'utilizzo di questa tecnica:

Per gli operatori

Riduzione delle difficoltà all'attacco e del rischio di errore;

Riduzione del tempo di emostasi.

Per i pazienti

Riduzione del dolore durante la venipuntura;

M iglioramento dell'estetica del braccio in sede di venipuntura della FAV.

Poiché questa esperienza si è rivelata sicuramente positiva abbiamo come obiettivo prossimo quello di introdur re altri pazienti con FAV di recente confezionamento.

\section{Infermieri del Centro Dialisi di Como}

Nel Centro Dialisi di Como dializziamo abitualmente circa 180 pazienti, articolati su 3 turni giornalieri, assistiti da 10 infermieri per turno.

N ell'aprile 2007, dopo una attenta valutazione e l'osservazione di esperienze in altri Centri, abbiamo introdotto nel nostro Centro la tecnica della puntura ad occhiello.

L'équipe di lavoro era composta inizialmente da 1 medico e da 3 infermieri i quali si sarebbero alternati nella sala e nei turni frequentati dai pazienti che sarebbero stati prescelti per la puntura ad occhiello.

Allo scopo è stata individuata una sala dialisi composta da 4 posti tecnici per un totale di 24 pazienti, ai quali è stato proposto un questionario iniziale le cui domande erano:

- Quanto incidesse il dolore durante la venipuntura.

- Quanto fosse prolungata l'emostasi allo stacco. 
Valutate le risposte, abbiamo proposto loro la tecnica della puntura ad occhiello.

Ai 24 pazienti abbiamo esposto i possibili vantaggi e svantaggi di tale procedura, di questi 17 hanno accettato.

Solo 2 pazienti avevano FAV native di recente confezionamento, le restanti sono state confezionate anche molti anni addietro.

Con la collaborazione del medico abbiamo individuato i punti di incannulazione, per alcuni ex novo (2) e per altri (22) mantenendo quelli già esistenti ma privi di tessuto cicatriziale.

In nessun caso l'incannulazione arteriosa e venosa avviene sullo stesso vaso.

Abbiamo seguito il protocollo che prevede per 8 sedute l'utilizzo di aghi taglienti per poi passare successivamente alI'uso degli aghi smussi (8 paz)

In alcuni casi ( 9 paz) si è dovuto riutilizzare l'ago tagliente per difficoltà alla puntura (effetto rimbalzo dell'ago).

All'inizio abbiamo riscontrato difficoltà nella rimozione delle croste e per il sanguinamento intradialitico in sede di inserzione dell'ago stesso (motivo per l'esclusione di 4 pazienti).

In un secondo tempo grazie all'acquisizione di maggiore esperienza e manualità nella procedura questo problema non si è più verificato.

Trascorsi circa tre mesi abbiamo sottoposto nuovamente un questionario ai 13 pazienti rimasti nella metodica per chiedere quali vantaggi avessero riscontrato con la nuova tecnica.

Tutti hanno risposto in modo positivo:

II dolore legato all'incannulazione era notevolmente ridotto, assente con l'utilizzo degli aghi smussi ( 6 pazienti) e

per tutti l'emostasi allo stacco risultava meno prolungata.

I vantaggi riscontrati dagli operatori:

- nessuna probabilità di errore all'incannulazione;

- riduzione del tempo di emostasi;

- a distanza di un anno le FAV (2 paz) di recente confez. non presentano aneurismi.

\section{Conclusioni}

Secondo noi è importante per I'utilizzo di questa tecnica scegliere FAV native recenti prive di aneurismi, lineari, con siti di puntura più facilmente reperibili, con cute più elastica e priva di tessuto cicatriziale in modo da ridurre tutti quegli effetti collaterali (lacerazioni, sanguinamento) che ci hanno portato ad escludere alcuni dei pazienti selezionati in questa prima fase.

Riteniamo comunque che questa metodica sia ottimale in piccoli Centri dove il turnover del personale infermieristico è più contenuto e quindi più attuabile a livello organizzativo, poiché, soprattutto durante la prima fase della metodica, è fondamentale che la venipuntura sia effettuata il più possibile dalla stessa mano.

\section{F. Cavatorta}

Direttore della Struttura Complessa di N efrologia e Dialisi, Ospedale di Imperia, ASL 1 imperiese

Dai due casi clinici descritti da M arisa Pegoraro emerge con evidenza che la tecnica ad occhiello introdotta recentemente in Italia merita una maggiore attenzione da parte del personale sanitario che lavora in dialisi.

Come giustamente viene sottolineato nella presentazione dei casi vi è quasi sempre nella maggior parte degli addetti ai lavori una particolare diffidenza verso tutto quello che è nuovo e/ o che contrasta con abitudini o convinzioni consolidate, tanto più quando la novità viene proposta da personale infermieristico particolarmente motivato e attento. $\mathrm{M}$ a come? Bisogna pungere la fistola sempre nella stessa sede e per di più con un ago non tagliente? $\mathrm{E}$ come la mettiamo con il rischio infettivo e il possibile sanguinamento dalla sede di puntura? $M$ a no, è stato insegnato che bisogna sempre spostare di circa $1 \mathrm{~cm}$ la sede di puntura da una volta all'altra! Perché cambiare e adottare una tecnica di dubbia affidabilità, meglio proseguire come si è sempre fatto in tutti questi anni di onorato servizio. 
Peccato che le cose non stiano realmente così!

L'esperienza che il Centro di I mperia sta accumulando dal giugno 2006 a oggi e che riguarda ben 8 pazienti, conferma invece quanto $\mathrm{M}$ arisa Pegoraro sta affermando con foga e passione.

In un caso molto simile al Caso Clinico 1, la tecnica ad occhiello è stata iniziata in un paziente in dialisi da 27 anni sempre con la stessa fistola artero-venosa distale radio-cefalica sx dietro sua esplicita richiesta dopo aver visto i risultati nei primi 4 pazienti in cui era stata adottata.

D opo una corretta informazione sulle indicazioni e finalità della metodica venne concordato con il paziente di iniziare la puntura ad occhiello anche per evitare il progressivo aumento di volume dei vari aneurismi presenti nell'avambraccio. Come nel Caso Clinico 2, anche noi abbiamo utilizzato la puntura ad occhiello in una fistola distale di recente confezionamento in un paziente con eritroder mia psoriasica; la scelta è stata determinata dalla difficoltà nell'eseguire la puntura a scala di corda per lo sviluppo modesto dell'accesso. Questa tecnica ha consentito di evitare probabili ematomi da puntura errata con possibile compromissione della sopravvivenza della fistola.

$\mathrm{N}$ on possiamo non condividere quanto riportato nelle conclusioni del Caso Clinico 1 dove si richiama la dovuta attenzione non solo all'aspetto estetico, ma soprattutto a quello del risparmio del patrimonio vascolare dei nostri pazienti e concludere con l'auspicio che la tecnica ad occhiello venga sempre più utilizzata in dialisi.

Ne deriverà un sicuro beneficio per il paziente il quale avvertirà molto meno dolore alla puntura, per l'accesso vascolare che vedrà maggiormente garantita la sua durata e per il personale sanitario che come dice M arisa Pegoraro potrà contribuire al benessere psico-fisico del proprio paziente.

\section{F. FIORINI}

\section{U.O. di Nefrologia e Dialisi Sanremo, ASL1 Imperiese}

La tecnica di venipuntura ad occhiello della fistola arterovenosa (FAV) nel paziente in emodialisi (HD) rappresenta una delle tante dimostrazioni di come la medicina in senso lato prosegua la sua continua evoluzione, la sua innovazione con la sistematica revisione di tecniche, protocolli ecc. atti a "migliorare" la prestazione fornita all'Assistito/ M alato/ Paziente/ Utente sulla base di quanto dettato dalla M edicina Basata sull'E videnza, ma anche come al contempo sussista una cer ta difficoltà nell'accettare le "novità" e a farle proprie quotidianamente.

$\mathrm{Nel}$ caso specifico per anni è stata consigliata la venipuntura con rotazione costante degli aghi (cosiddetta "puntura a scala di corda") e la ricerca continua di nuovi siti di venipuntura sullo stesso paziente emodializzato al fine di evitare da un lato emorragie post dialisi per formazione di "brecce" importanti e dall'altro il formarsi di aneurismi della FAV, sede di importante patologia vascolare (calcificazioni, trombosi, infezioni, stenosi) : nella pratica clinica di sala dialisi si assisteva invece a una venipuntura a sito pressoché costante sia per il lato arterioso che per quello venoso a causa della volontà sinergica di pazienti e infermieri che rispettivamente non vogliono sentire male (leggi subire "nuovi buchi") e hanno paura di sbagliare (mancata venipuntura e necessità di un nuovo tentativo).

È comunque strano/ singolare che una tecnica che trova la sua origine nei primi anni Settanta, proposta addirittura da un "cer to" dottor Twardowsky, sia stata ripresa solo nel 2004 e riproposta con discreto vigore solo negli ultimi due/ tre anni non solo in Italia, ma anche in buona parte dell'E uropa. Sicuramente diversi sono i fattori che hanno determinato questo "nuovo" interesse. La sempre maggior attenzione per la persona "malata" da parte di tutti gli operatori sanitari ha creato i presupposti per la ricerca di nuove soluzioni atte a risparmiare il più possibile i vasi del paziente uremico in HD: infatti la FAV non solo è il migliore accesso vascolare, il suo buon funzionamento rappresenta la conditio sine qua non per ottenere un'HD efficace.

Risulta evidente come tale impostazione metodologica sia il frutto del coinvolgimento collaborativo di più professionalità sanitarie e come la stessa cosa debba ricercarsi in fase di istruzione fra le medesime professionalità. Ci si riferisce a decisioni fondamentali quali ad esempio la scelta dei siti di venipuntura che deve prevedere pressoché in tutti i casi uno studio ecografico accurato di tutta la FAV, soprattutto se si tratta di una "vecchia" FAV, al fine di individuare siti che possano prevedere una lunga utilizzazione.

Le professionalità infermieristiche sono e saranno sempre più le depositarie della storia e della "vita" della FAV (e non solo di questa!) di ogni singolo paziente, in considerazione della sempre maggiore area di autonomia lavorativa a loro attribuita e dalla concomitante riduzione del numero del personale medico cui si è assistito in que- 
st'ultimo decennio. Per il personale infermieristico si tratta in alcuni casi di superare una certa prudenza/ titubanza storico/ culturale nell'introdurre nuove tipologie gestionali dell'accesso vascolare, nella operatività sempre più autonoma di varie problematiche con assunzione di maggiori responsabilità anche cliniche, come ad esempio quella della rimozione delle croste. In questo caso, ad esempio, non deve essere presente solo una discreta manualità, ma anche una continua motivazione individuale nell'insegnare al paziente ad avere una costante cura e igiene della propria FAV, ricordandogli sempre di lavare l'arto interessato prima della seduta dialitica (a domicilio o nel Centro stesso) sia per ammorbidire le croste, ma anche per prestare una prima disinfezione dell'arto.

Sicuramente solo la collaborazione e l'assunzione piena di responsabilità di tutti gli operatori sanitari potranno permettere un continuo, lento ma costante miglioramento della qualità delle cure e dell'assistenza.

\section{LOMBARDI}

N efrologia e Dialisi, Ospedale S.M . Annunziata, Azienda Sanitaria di Firenze, Firenze

Tutto ciò che si può dire lo si può dire chiaramente.

Su ciò di cui non si può parlare si deve tacere.

L. Wittgenstein, in Tractatus logico-philosophicus

Perché aprire un numero del Giornale di $T N \& D$ scrivendo su una tecnica di venipuntura dell'accesso vascolare per emodialisi?

A quarant'anni dalla più geniale "invenzione" per la connessione del paziente al circuito extracorporeo (la fistola artero-venosa) (1) ci si può attendere ancora qualcosa di nuovo in tema di accessi vascolari? Soprattutto al riguardo della tecnica della loro venipuntura?

Chi opera in un Centro emodialisi sa bene che ancor oggi la sostenibilità di una tecnica così importante, come quella sostitutiva la funzione renale, può venir messa in forse dall'esaurimento del patrimonio vascolare del paziente... e, come direbbe Ludwig W ittgenstein, ... sino a che un solo essere al mondo deve morire d'uremia per mancanza delI'accesso vascolare, tutto il mondo sta morendo di uremia... (2).

D obbiamo allora augurarci ogni buona nuova anche su questo argomento. Cer to che definire una novità la tecnica di puntura dell'accesso vascolare a sito costante (detta anche ad "occhiello o buttonhole technique") sarebbe veramente mendace. Basti pensare, come fa notare M arisa Pegoraro in questo stesso numero, che Twardowsky e Kubara (3) hanno proposto la tecnica già negli anni Settanta.

Possibile che a quasi quarant'anni di distanza si possa tranquillamente presentare alla maggior parte degli addetti ai lavori la puntura ad occhiello come una novità? Possibile, perché tale tecnica ha avuto una inspiegabile diffusione limitata nonostante le poche pubblicazioni sull'argomento siano tutte favorevoli all'uso della tecnica.

Ci sono alcune credenze da sfatare come il fatto che la tecnica sia adatta solo a FAV "vergini" o con breve tratto pungibile o per la dialisi domiciliare o notturna. Viceversa vi sono ormai dimostrazioni che tale tecnica di venipuntura prevenga la formazione delle dilatazioni aneurismatiche, riduca gli ematomi, le punture dolorose, quelle er rate e la ricerca di nuovi siti di puntura, per cui anche il tempo dedicato dal personale all'incannulamento (4). Per contro per lo meno nella fase di creazione del tunnel l'operatore dovrebbe esser sempre lo stesso e ciò porta qualche difficoltà organizzativa.

\section{Bibliografia}

1. Brescia MJ, Cimino JE, Appel K, Hurwich BJ. Chronic hemodialysis using venipuncture and a surgically created arteriovenous fistula. N Engl J M ed 1966; 275(20): 1089-92.

2. M aiorca R, M aggiore Q, M ordacci R, Tonini E, Biagi E, Bissoni G. Ethical problems in dialysis and transplantation. Nephrol Dial Transplant 1996; 11[S9]: 100-12.

3. T wardowsky Z, Kubara H. Different sites versus constant site of needles insertion into arteriovenous fistulas for treatment by repeated dialysis. Dial Transplant 1979; 8: 978-80.

4. Ball LK. Buttonhole technique for arteriovenous fistula cannulation. Nephrology Nursing J 2006; 33(3): $299-304$. 